\title{
Microarray of surface-exposed proteins of rickettsia heilongjiangensis for serodiagnosis of Far-eastern spotted fever
}

\author{
Yong $\mathrm{Qi}^{\dagger}$, Wenping Gong ${ }^{\dagger}$, Xiaolu Xiong, Jiafu Jiang, Yawei Wang, Jun Jiao, Changsong Duan and Bohai Wen*
}

\begin{abstract}
Background: Far-eastern spotted fever (FESF) is an important emerging infectious disease in Northeast Asia. The laboratory diagnosis of FESF in hospitals is mainly based on serological methods. However, these methods need to cultivate rickettsial cells as diagnostic antigens, which is both burdensome and dangerous.

Methods: Eleven surface-exposed proteins (SEPs) were identified in our previous study and their recombinant proteins ( $r S E P s)$ fabricated on a microarray were serologically analyzed with seventeen paired sera from patients suffered from FESF in this study.

Results: All the rSEPs showed sensitivities of between 53\% and $82 \%$ to acute-phase sera and of between $65 \%$ and $82 \%$ to convalescent-phase sera, and all the rSEPs except rRplA showed specificities of between $80 \%$ and $95 \%$. The combination assay of two, three, or four of the four rSEPs (rOmpA-2, rOmpB-3, rRpsB, and rSdhB) showed better sensitivities of between $76 \%$ and $94 \%$ to the acute-phase sera or between $82 \%$ and $100 \%$ to the convalescent-phase sera and acceptable specificities of between $75 \%$ and $90 \%$.
\end{abstract}

Conclusions: Our results suggest that the four rSEPs are more likely candidate antigens for serological diagnosis of FESF.

Keywords: Far-eastern spotted fever, Rickettsia heilongjiangensis, Protein microarray, Serological diagnosis

\section{Background}

Rickettsia heilongjiangensis, a spotted fever group (SFG) rickettsia isolated from ticks in 1983 in Heilongjiang Province of China [1], is the causative agent of Far-eastern spotted fever (FESF). FESF has been considered as an important emerging infectious disease in Northeast Asia for this rickettsiosis has been diagnosed in Northeast of China [2], east-Siberian and far-eastern regions of Russia [3,4], and Japan [5]. Most of the patients naturally infected by $R$. heilongjiangensis had fever, chills, headache, dizziness, myalgias, arthralgias, and anorexia after an incubation period of 4 to 7 days, and later most of the patients appeared with a macular or maculopapular rash at the site of tick attachment and lymphadenopathy regional to the eschar [4]. Almost half of the patients had hepatomegaly accompanied

\footnotetext{
* Correspondence: bohaiwen@sohu.com

${ }^{\dagger}$ Equal contributors

State Key Laboratory of Pathogen and Biosecurity, Beijing Institute of Microbiology and Epidemiology, 20 Dong-Dia-Jie Street, Fengtai, Beijing, China
}

\section{Ciomed Central}

with an increased alanine aminotransferase and/or aspartate aminotransferase activity [4]. In a murine model, $R$. heilongjiangensis caused severe systemic infection with lesions in multiple organs (liver, lung, and brain) [6].

So far, the laboratory diagnosis of rickettsioses in hospitals is mainly based on serological methods although cell culture and molecular tools like PCR or real-time PCR are applied [7]. Immunofluorescence assay (IFA) is the gold standard and is used as a reference technique in most laboratories [7]. A gold diagnostic standard for rickettsioses, IFA followed by real-time PCR, has been built in the French National Reference Center (FNRC) [8]. However, IFA needs to cultivate rickettsial cells as diagnostic antigens, which is both burdensome and dangerous.

Some efforts have been focused on screening rickettsial proteins as serodiagnostic antigens of rickettsioses $[9,10]$. Kowalczewska et al. characterized 20 rickettsial recombinant proteins by enzyme-linked immune sorbent assay (ELISA) with sera from patients infected by $R$. typhi or $R$. 
conorii [10], in which, many surface-exposed proteins (SEPs) like Adr2, Omp1, PLD, RickA, Sca1, Sca10, and Sca13 were used. In fact, many SEPs have been found to be suitable as diagnostic antigens, such as a $56 \mathrm{kDa}$ outer membrane protein in detection of Orientia tsutsugamushi infection [11,12] and a surface protein Pap31 in detection of Bartonella bacilliformis infection [13]. These findings indicate that SEPs are more likely to be diagnostic candidates. In our previous study, 24 SEPs of $R$. heilongjiangensis were identified and their recombinant proteins (rSEPs) fabricated on a microarray were serologically analyzed and eleven of them were recognized as major seroreactive proteins and potential candidate antigens for serological diagnosis of FESF by sera from mice experimentally infected with $R$. heilongiangensis [9]. Also, these rSEPs in microarray assay showed a higher specificity in recognizing $R$. heilongjiangensis-infected mouse sera compared with that in ELISA [9]. In the present study, these rSEPs fabricated on a protein microarray were assayed with paired sera from FESF patients during the acute and convalescent phase.

\section{Methods}

\section{Patient sera}

FESF was diagnosed in patients by PCR using whole blood [14] as well as clinical symptoms consistent with tick-bite fever, multiple inoculation eschars and cutaneous rash in hospital. IgG antibody titers of patient sera were determined by IFA with $R$. heilongjiangensis antigen as described previously [9]. Each case of FESF was confirmed by a single serum with the specific IgG titer of $\geq 1: 128$ or the paired sera with a fourfold or greater increase of the specific IgG titers. The paired sera were collected from 17 patients suffered from FESF during the acute and convalescent phase. The acute-phase sera were collected from the patients at the date of onset of illness, and the convalescent-phase sera were collected from the same patients approximately two weeks after the first sampling. Also, 20 sera, collected from acute febrile patients with uncertain diagnoses and their titers of IgG antibodies to $R$. heilongjiangensis being determined to be less than or equal to 1:8 in IFA, were used as negative control or as reference sera to assess diagnostic specificity of the microarray assay in this study.

All of the patient sera were obtained from a hospital in northeast China. The serum samples of patients were collected as part of the routine management of patients without any additional sampling. All patients gave their informed consent and all patient data were deidentified. The Institutional Review Board of the Beijing Institute of Microbiology and Epidemiology approved the research involving human materials.

\section{Preparation of recombinant proteins}

Eleven rSEPs of $R$. heilongjiangensis, including rGroEL, rOmpA-2, rOmpB-3, rPrsA, rRplA, rRplY, rRpsB, rSdhB,
rSurA, rYbgF, and rRh054_02285, were used in the present study. The preparation and purification of these recombinant proteins were described in our previous study $[9,15]$.

\section{Immunoblotting assay}

The purified rSEPs were immunoblotted with the paired sera from one patient with FESF. Briefly, rSEPs separated by SDS-PAGE were transferred to polyvinylidene difluoride (PVDF) membrane. The PVDF membrane was blocked with $1 \%[\mathrm{w} / \mathrm{v}]$ bovine serum albumin (BSA) in phosphate buffer saline (PBS, containing $8.1 \mathrm{mM} \mathrm{Na} \mathrm{HPO}_{4}, 1.9 \mathrm{mM}$ $\mathrm{NaH}_{2} \mathrm{PO}_{4}$, and $154 \mathrm{mM} \mathrm{NaCl}$ ) at $\mathrm{pH} 7.4$ overnight. Then, rSEPs on the PVDF membrane were incubated with the acute- or convalescent-phase serum (1:250 dilution) that was previously neutralized with $E$. coli lysate $(5 \mathrm{mg} / \mathrm{ml})$ for $1 \mathrm{~h}$. After three washes in PBST ( $\mathrm{pH} 7.4$ PBS containing $0.05 \%$ [v/v] Tween 20), the PVDF membrane was incubated with horseradish peroxidase (HRP)-conjugated goat antihuman IgG (1:5 000 dilution; Beijing CoWin Biotech, Beijing, China) for $1 \mathrm{~h}$. Following an additional three washes in PBST, the PVDF membrane was developed using a diaminobenzidine (DAB) kit (Boster, Wuhan, China).

\section{Fabrication of protein microarray}

Each of the purified rSEPs diluted in PBS to a concentration of $0.3 \mathrm{mg} / \mathrm{ml}$ was printed on epoxy slides (CapitalBio, Beijing, China) in 5 replicate spots as described previously [16]. Human IgG with serial dilutions (2.5, 5, 10 and $20 \mu \mathrm{g} / \mathrm{ml}$ ) was used to fit the internal calibration curves or as positive controls. BSA in PBS or lysate of E. coli cells transformed with pET-32a plasmids at a concentration of $0.3 \mathrm{mg} / \mathrm{ml}$ was used as negative controls [16]. For quality control, the microarray slides were incubated with mouse anti-His tag IgG-Cy5 (SBA, Birmingham, AL) and the fluorescence intensity (FI) of each protein on the slides was scanned by GenePix Personal 4100A (Molecular Devices, Sunnyvale, CA) and analyzed by GenePix Pro 6.0 software (Molecular Devices, Sunnyvale, CA) [16]. Proteins with a signal-to-background ratio over 3.0 were used for further analysis [16].

\section{Analysis of proteins on microarrays by patient sera}

The rSEPs on the microarray slide were probed using patient sera according to previous descriptions [9]. Briefly, the microarray slide was blocked with $1 \%[\mathrm{w} / \mathrm{v}] \mathrm{BSA}$ in PBS overnight. Then each well on the slide was incubated with $50 \mu \mathrm{l}$ of each patient serum (1:50 dilution) that were previously neutralized with $E$. coli lysate $(5 \mathrm{mg} / \mathrm{ml})$ for $1 \mathrm{~h}$ [16]. After six washes in PBST, the microarray slide was developed by incubation with goat-anti human IgG-Cy5 (SBA) (1:500 dilution) for $1 \mathrm{~h}$. Following an additional five washes in PBST and a final wash in deionized water, the air dried microarray slide was scanned with a GenePix 
Personal 4100A scanner and the scanned images were analyzed by GenePix Pro 6.0. The FI value of each protein was calculated by averaging the FI values of five replicate spots, which had been background-subtracted [9].

\section{Microarray data analysis}

Human IgG dose-FI value curves were fitted with linear regression analysis using GraphPad Prism 5 software (GraphPad Software, Inc., San Diego, CA). The relative amounts of specific IgG (RASIgG) to individual rSEPs in each serum were determined by interpolating the calculated FI value with the IgG internal calibration curve [17].

The cutoff value for individual rSEPs in the microarray assay was generated as described previously using Youden's index [13]. The reaction was considered positive if the RASIgG to one rSEP in any of the patient sera was higher than the cutoff value.

\section{Statistical analysis}

The log-transformed IgG titers to $R$. heilongjiangensis in IFA and the numbers of rSEPs recognized by FESF patient sera in microarray assay were analyzed for potential correlations by linear regression using GraphPad Prism 5 software.

The IgG titer of the convalescent-phase serum divided by the IgG titer of the acute-phase serum was calculated as the increased titer for each paired sera. The increase in RASIgG to each rSEP in each paired sera was calculated as follows: The increased RASIgG = RASIgG to one rSEP in the convalescent-phase serum/RASIgG to the same rSEP in the acute-phase serum.

In paired sera, the correlation between the increase in IgG titers (log transformed) to $R$. heilongjiangensis and the increase in RASIgG to individual rSEPs was analyzed by linear regression using GraphPad Prism 5 software.

\section{Results}

IgG titers of sera determined by IFA

The IgG titers of 17 paired sera from FESF patients were determined by IFA with $R$. heilongjiangensis antigen. Fourteen of these paired sera showed a fourfold or greater rise in specific IgG titers and the other 3 paired sera showed high specific IgG titers greater than or equal to 1:128 (Table 1).

\section{Immunoblotting assay}

Eleven rSEPs of $R$. heilongjiangensis were immunoblotted by the acute- or convalescent-phase serum from one of FESF patients (patient 8 in Table 1). As shown in Figure 1, seven rSEPs (rGroEL, rOmpA-2, rRh054_02285, rRplA, rRpsB, rSurA, and rYbgF) were recognized strongly by both acute- and convalescent-phase serum and the rest were recognized weakly. Seven rSEPs (rGroEL, rOmpA-2, rPrsA, rRplA, rRpsB, rSdhB, and rYbgF) showed a stronger
Table 1 The IgG titers of paired sera from patient suffered from FESF in IFA

\begin{tabular}{|c|c|c|c|}
\hline Patients no. & $\begin{array}{l}\text { Acute-phase } \\
\text { serum sample }\end{array}$ & $\begin{array}{l}\text { Convalescent-phase } \\
\text { serum sample }\end{array}$ & $\begin{array}{l}\text { Diagnostic } \\
\text { criteria }\end{array}$ \\
\hline 1 & 128 & 512 & Fourfold increase \\
\hline 2 & 64 & 1024 & Fourfold increase \\
\hline 3 & 256 & 512 & IgG titer $\geq 128$ \\
\hline 4 & 64 & 256 & Fourfold increase \\
\hline 5 & 32 & 512 & Fourfold increase \\
\hline 6 & 128 & 1024 & Fourfold increase \\
\hline 7 & 128 & 1024 & Fourfold increase \\
\hline 8 & 256 & 512 & IgG titer $\geq 128$ \\
\hline 9 & 32 & 512 & Fourfold increase \\
\hline 10 & 256 & 256 & IgG titer $\geq 128$ \\
\hline 11 & 64 & 1024 & Fourfold increase \\
\hline 12 & 64 & 1024 & Fourfold increase \\
\hline 13 & 64 & 512 & Fourfold increase \\
\hline 14 & 128 & 1024 & Fourfold increase \\
\hline 15 & 128 & 1024 & Fourfold increase \\
\hline 16 & 64 & 512 & Fourfold increase \\
\hline 17 & 64 & 1024 & Fourfold increase \\
\hline
\end{tabular}

staining reaction with the convalescent-phase serum than with the acute-phase serum.

\section{Quality control of protein microarray}

For quality control, the microarray slides printed with rSEPs were incubated with mouse anti-His tag IgG-Cy5 (SBA, Birmingham, AL) and scanned for their FI values. The coefficient of variations $(\mathrm{CV})$ was calculated as the SD of the FI value for each SEP divided by the average FI value. As a result, the within-slide CV $(n=6)$ and between-slide $\mathrm{CV}(\mathrm{n}=6)$ of individual rSEPs on the microarray ranged from $8 \%$ to $18 \%$.

\section{Sensitivity and specificity of rSEPs in microarray assay}

Internal calibration curve of the microarray was generated by probing a serial dilution of human IgG solution with goat-anti human IgG-Cy5 and the FI value of BSA probed with goat-anti human IgG-Cy5 was set as the first point of the internal calibration curve. Linear regression analysis revealed that all the calibration curves gave the good correlation coefficients $\left(\mathrm{r}^{2}\right)$ ranging from 0.967 to 0.997 (Figure 2).

Eleven rSEPs on the microarray were probed with the patient sera. As a result (Table 2), all the rSEPs showed sensitivities of between $53 \%$ and $82 \%$ in recognizing acute-phase sera and of between $65 \%$ and $82 \%$ in recognizing convalescent-phase sera, and all the rSEPs except rRplA showed specificities of between $80 \%$ and $95 \%$. Four rSEPs (rOmpA-2, rRplA, rRpsB, and rSdhB) showed 


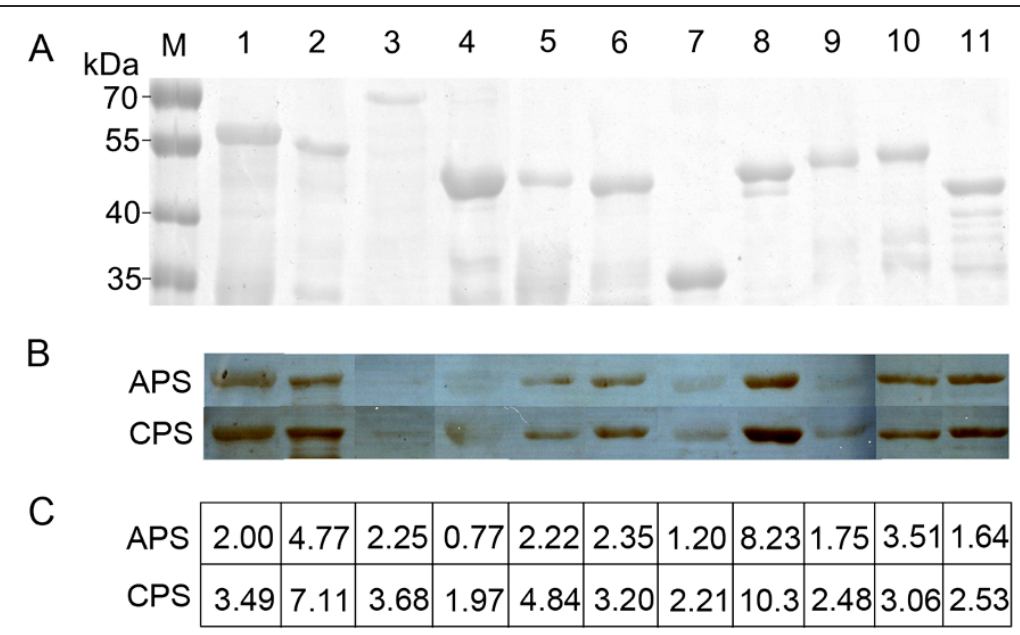

Figure 1 SDS-PAGE and immunoblotting analysis of the purified rSEPs. Eleven rSEPS were analyzed with SDS-PAGE (A) and immunoblotted with acute-phase sera (APS) or convalescent-phase sera (CPS) of patient 8 (B). The relative amount of specific antibodies to individual rSEPs in APS or CPS of patient 8 was calculated in microarray assay (C). Lanes 1 to 11 refer to rGroEL, rOmpA-2, rOmpB-3, rPrsA, rRh054_02285, rRplA, rRplY, rRpsB, rSdhB, rSurA, and rYbgF, respectively. Lane M refers to protein markers and their relative molecular masses are indicated in $\mathrm{kDa}$ on the left.

sensitivities of $\geq 71 \%$ in recognizing both acute- and convalescent-phase sera. Also the summary of sensitivities of each protein to both acute- and convalescent-phase sera and specificity of each protein was calculated to generally evaluate its ability as a candidate antigen for diagnosis of FESF. As a result (Table 2), four rSEPs (rOmpA-2, rOmpB3 , rRpsB, and rSdhB) scored higher than the rest.

\section{Relationship between specific lgG titers of sera and seroreactivity of $r S E P s$}

Linear regression analysis revealed a significant positive correlation between the log-transformed IgG titers to $R$. heilongjiangensis in all the patient sera and the numbers of proteins recognized by these sera (Figure $3, \mathrm{r}^{2}=$ 0.5381, $P<0.0001, \mathrm{n}=54$ ).

In addition, linear regression analysis revealed that significant correlations between the increase in the log-

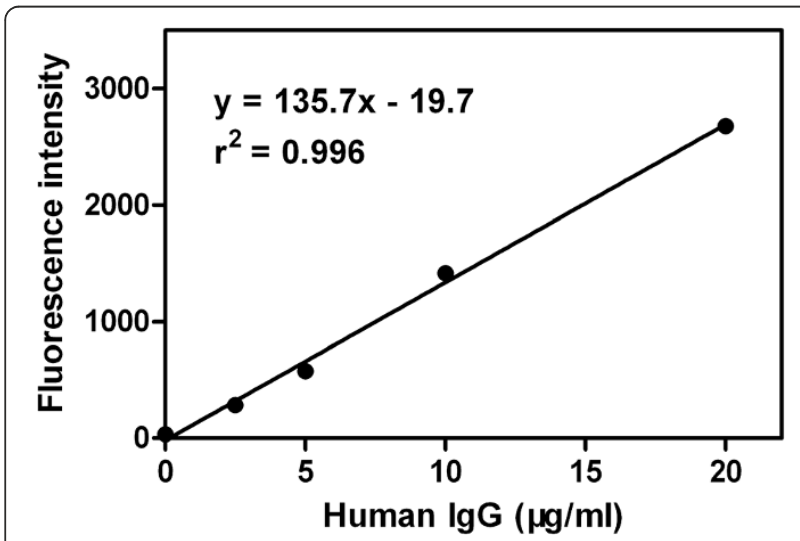

Figure 2 One example of the internal calibration curves generated using serial dilutions $(0,2.5,5,10$ and $20 \mu \mathrm{g} / \mathrm{ml})$ of human IgG probed with goat-anti human IgG-Cy5. transformed IgG titers to $R$. heilongjiangensis for paired sera and the rising in RASIgG to rOmpA-2 $\left(\mathrm{r}^{2}=0.2621\right.$, $P=0.0356)$ or $\mathrm{rRpsB}\left(\mathrm{r}^{2}=0.2838, P=0.0277\right)$ in these sera (Table 3).

\section{Discussion}

In our previous study [9], eleven SEPs of $R$. heilongjiangensis were recognized as major seroreactive antigens and potential candidate antigens for serological diagnosis of FESF in microarray assay with $R$. heilongjiangensis-infected mouse sera. In the present study, their recombinant proteins fabricated on a microarray were

Table 2 The sensitivity and specificity of individual rSEPs on microarray probed with the acute-phase sera $(n=17)$ or convalescent-phase sera $(n=17)$

\begin{tabular}{|c|c|c|c|c|}
\hline \multirow[b]{2}{*}{ Proteins } & \multicolumn{2}{|c|}{ Sensitivity (\%) } & \multirow{2}{*}{$\begin{array}{c}\text { Specificity } \\
(\%)\end{array}$} & \multirow{2}{*}{$\begin{array}{c}\text { Summary* } \\
(\%)\end{array}$} \\
\hline & $\begin{array}{l}\text { Acute- } \\
\text { phase }\end{array}$ & $\begin{array}{c}\text { Convalescent- } \\
\text { phase }\end{array}$ & & \\
\hline rGroEL & 65 & 65 & 80 & 209 \\
\hline rOmpA-2 & 71 & 82 & 85 & 238 \\
\hline rOmpB-3 & 65 & 82 & 90 & 237 \\
\hline rPrsA & 53 & 76 & 95 & 224 \\
\hline rRh054_02285 & 71 & 65 & 90 & 225 \\
\hline rRplA & 71 & 82 & 65 & 218 \\
\hline rRplY & 65 & 82 & 85 & 232 \\
\hline rRpsB & 71 & 71 & 95 & 236 \\
\hline rSdhB & 82 & 76 & 90 & 249 \\
\hline rSurA & 59 & 82 & 90 & 231 \\
\hline rYbgF & 65 & 65 & 90 & 219 \\
\hline
\end{tabular}

*The summary of sensitivities of each protein to both acute- and convalescent-phase sera and specificity of each protein was calculated to evaluate its ability as a candidate antigen for diagnosis of FESF. 


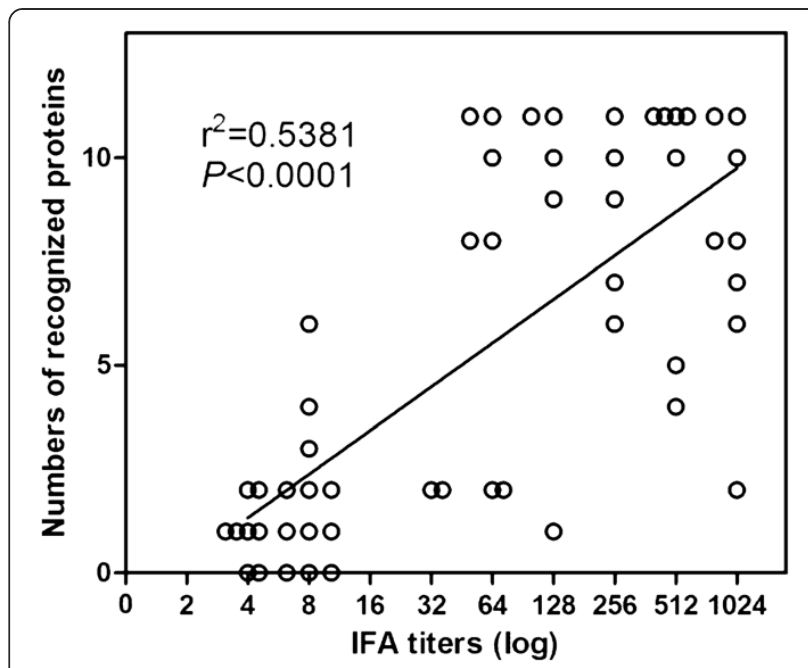

Figure 3 Linear regression analysis to examine potential relationships between the IgG titers of FESF patient sera $(n=54)$ and the numbers of rSEPs recognized by these sera.

The IgG titer of each serum in IFA was log transformed. The analysis was conducted using GraphPad Prism 5 software (GraphPad Software, Inc., San Diego, CA).

assayed with paired sera from FESF patients so as to identify potential candidate antigens for serological diagnosis of FESF, as well as to explore the kinetic change of the specific antibodies to individual SEPs in FESF patients.

Firstly, these rSEPs were immunoblotted by paired sera from one FESF patient, and most of them showed a stronger reaction with the convalescent-phase serum than with the acute-phase serum, which suggested that more specific antibodies to these SEPs appeared in the convalescentphase serum. This could not be quantitatively determined

Table 3 Linear regression analysis to examine potential relationships between the increased IgG titers to $R$. heilongjiangensis and the increased IgG level to individual rSEPs in paired sera

\begin{tabular}{lcc}
\hline Protein name & Coefficients of correlations $\left(\mathbf{r}^{\mathbf{2}}\right)$ & $\boldsymbol{P}$ value \\
\hline rGroEL & 0.09251 & 0.2353 \\
rOmpA-2* & 0.2621 & 0.0356 \\
rOmpB-3 & 0.01372 & 0.6544 \\
rPrsA & 0.1329 & 0.1502 \\
rRh054_02285 & 0.002353 & 0.8533 \\
rRplA & 0.06482 & 0.3241 \\
rRplY & 0.003742 & 0.8156 \\
rRpsB* & 0.2838 & 0.0277 \\
rSdhB & 0.005894 & 0.7696 \\
rSurA & 0.05963 & 0.3449 \\
rYbgF & 0.04017 & 0.4405 \\
\hline
\end{tabular}

*Statistically significant $(P<0.05)$ associations are marked. in the immunoblotting assay. However, the reactivity of each rSEP with individual sera was quantitatively determined by the microarray assay. In addition, the FI value of each protein probed with individual sera was interpolated with the calibration curve, which minimized variability in this quantitative determination so as to improve the within-slide and between-slide analytical precision.

The individual rSEPs were analyzed by both immunoblot and microarray assay using the paired sera from patient 8. All of the rSEPs except OmpB-3 and YbgF, to which the RASIgG were higher in microarray, showed a stronger staining reaction in immunoblot assay (Figure 1B,C). The exception may be due to the different states of OmpB-3 and YbgF existing in different assays. OmpB-3 was stained very lightly in immonoblot while the RASIgG in the serum detected with microarray was big, which might be due to the tertiary structure of OmpB-3 on the microarray slide that might have exerted a steric effect to promote non-specific absorption of IgG from the sera, one effect that would not apply to the denatured OmpB-3 in the immunoblot assay. YbgF was stained strongly in immunoblot assay while the RASIgG in microarray assay was small. YbgF was denatured and might provide more epitopes to bind the specific antibodies in immonoblot assay, while it maintained its native structure and might provide less epitopes to bind the specific antibodies in the microarray assay.

In this microarray assay, only $5 \%$ to $20 \%$ of reference sera from the acute febrile patients without antibodies to $R$. heilongiiangensis reacted positively to individual rSEPs except rRplA, suggesting these rSEPs had a good specificity. The cross-reaction might be caused by the conservative SEPs such as the ribosomal protein RplA and patients from whom the reference sera were collected have suffered from other infection caused by bacteria which shared the conservative SEPs with $R$. heilongiiangensis. All of these rSEPs gave a sensitivity of over $65 \%$ to the convalescent-phase sera from FESF patients while five rSEPs (rOmpA-2, rOmpB-3, rRplA, rRplY, and rSurA) had a higher sensitivity of $82 \%$ to them. However, only rSdhB had a higher sensitivity of $82 \%$ to the acute-phase sera from FESF patients and the other rSEPs gave sensitivities of only between $53 \%$ and $71 \%$ to them.

The summary of sensitivity and specificity was calculated and four rSEPs (rOmpA-2, rOmpB, rRpsB, and rSdhB) had relatively higher scores. Also we found that the four rSEPs could recognize $63 \%, 63 \%, 74 \%$, and $85 \%$ of the 19 FESF patient sera with lower IFA titer of $\leq 256$ (2, 7, 5 , and 5 of these sera have IFA titers of $32,64,128$, and 256, respectively), respectively. When combination analysis of the data resulting from two, three, or four of the four rSEPs was performed (Table 4), and the patient was diagnosed as having FESF if the serum sample from him or her is positively recognized by at least one of the rSEPs, 


\begin{tabular}{|c|c|c|c|}
\hline \multirow[b]{2}{*}{ Proteins } & \multicolumn{2}{|c|}{ Sensitivity (\%) } & \multirow{2}{*}{$\begin{array}{c}\text { Specificity } \\
\text { (\%) }\end{array}$} \\
\hline & $\begin{array}{l}\text { Acute- } \\
\text { phase }\end{array}$ & $\begin{array}{l}\text { Convalescent- } \\
\text { phase }\end{array}$ & \\
\hline rOmpA-2\&rOmpB-3 & 82 & 94 & 80 \\
\hline rOmpA-2\&rRpsB & 76 & 94 & 80 \\
\hline rOmpA-2\&rSdhB & 88 & 94 & 80 \\
\hline rOmpB-3\&rRpsB & 82 & 94 & 90 \\
\hline rOmpB-3\&rSdhB & 88 & 94 & 80 \\
\hline rRpsB\&rSdhB & 82 & 82 & 85 \\
\hline rOmpA-2, rOmpB-3\&rRpsB & 88 & 100 & 80 \\
\hline rOmpA-2, rOmpB-3\&rsdhB & 94 & 100 & 75 \\
\hline rOmpA-2, rRpsB\&rSdhB & 88 & 94 & 75 \\
\hline rOmpB-3, rRpsB\&rSdhB & 88 & 94 & 80 \\
\hline rOmpA-2, rOmpB-3, rRpsB\&rSdhB & 94 & 100 & 75 \\
\hline
\end{tabular}

better sensitivities of between $76 \%$ and $94 \%$ to the acutephase sera or between $82 \%$ and $100 \%$ to the convalescentphase sera and acceptable specificities of between $75 \%$ and $90 \%$ were obtained. Our results suggest that the remarkable variation in immune recognition patterns for FESF require multi-antigen combination to cover the different antibody responses and thus achieve the highest possible test sensitivity. Serological tests are the easiest methods for the diagnosis of rickettsiosis but seroconversion is usually detected 7-15 days after disease onset [18]. Our combination assay could recognize as many as $94 \%$ of the acute-phase sera and hopefully diagnose FESF rapidly at the early stage of infection. Therefore, the four rSEPs may be considered as more likely candidate antigens for the serological diagnosis of FESF, especially rSdhB, with its sensitivity of $82 \%$ to the acute-phase sera and $76 \%$ to the convalescent-phase sera with specificity of $90 \%$. Furthermore, refinement of the production of fusion molecules comprised of these SEPs and the reaction conditions of microarray assay described herein may lead to improve the sensitivity and specificity for the serodiagnosis of FESF. Epitopes in these proteins can be predicted using bioinformatics method and synthesized to evaluate their ability of diagnosis of FESF. Then the molecules fused with different combination of the selected epitopes may show better sensitivities and specificities.

All of these rSEPs except rRh054_02285 and rSdhB probed with the convalescent-phase sera gave the same sensitivity or a higher sensitivity than probed with the acute-phase sera. This is not unexpected since the convalescent-phase sera had higher titers of antibodies to $R$. heilongjiangensis. We noticed that RASIgG to
rRh054_02285 or rSdhB did decrease when probed with paired sera from some patients, indicating the specific IgG level to these proteins decreased quickly in some of these FESF patients during the convalescent phase.

Linear regression analysis revealed a significant positive correlation between the log-transformed specific IgG titers of FESF patient sera in IFA and the numbers of rSEPs recognized by these patient sera in microarray assay (Figure $3, P<0.0001$ ). This indicated that specific IgG levels to individual SEPs might contribute to the specific IgG titers to $R$. heilongjiangensis in FESF patient sera since they were major seroreactive antigens of $R$. heilongjiangensis. Moreover, the increase in IgG titers to $R$. heilongjiangensis for paired sera and the rising in RASIgG to rOmpA-2 or rRpsB in these sera were significantly correlated (Table $3, P<0.05$ ), indicating that antibodies to OmpA-2 or RpsB contributed more to the increase in IgG titers to $R$. heilongjiangensis than antibodies to other SEPs.

In the present study, the number of paired patient sera tested was small, which may influence the sensitivities and specificities of these rSEPs. Detection of specific IgM antibody to individual rSEPs might improve the sensitivity to acute-phase sera of FESF patients and unfortunately some paired patient sera were not enough to do this test. Therefore, it is necessary to get more serum samples of FESF patients for this microarray assay in the future.

\section{Conclusions}

In conclusion, the eleven SEPs were serologically characterized with paired sera from FESF patients, and four rSEPs (rOmpA-2, rOmpB-3, rRpsB, and rSdhB) are more likely candidate antigens for the serological diagnosis of FESF. In addition, an optimized microarray composed with the four rSEPs may give an acceptable sensitivity for serological diagnosis of FESF during both the acute and convalescent phase.

\section{Competing interests}

The authors declare that they have no conflict of interest.

\section{Authors' contributions}

$Y Q$ and WG carried out the experiments, data analyses and drafted the manuscript. XX assisted the analysis of data; JJ and YW provided the patient sera and helped to draft the manuscript; $J J$ and CD participated in its design and helped to draft the manuscript; BW designed the experiments and revised the manuscript. All authors read and approved the final manuscript.

\section{Acknowledgments}

This work was supported by a grant (2010CB530200/2010CB530205) from the National Basic Research Program of China and a grant (2013ZX10004803) from the Natural Science and Technology Major Project of China.

Received: 14 April 2014 Accepted: 13 June 2014

Published: 17 June 2014 


\section{References}

1. Lou D, Wu YM, Wang B, Lui GD, Li JZ, Wang W, Han YF: A new member of the spotted fever group of rickettsiae-Rickettsia heilongjiangensis. Chin $J$ Microbiol Immunol 1985, 5:250-253.

2. Wu Y, Zhang Z, Wang H, Yang Q, Feng L, Wang J: Investigation on the epidemiology of Far-East tick-borne spotted fever in the Northeastern area of China. Chin J Epidemiol 2008, 29(12):1173-1175.

3. Shpynov SN, Fournier PE, Rudakov NV, Samoilenko IE, Reshetnikova TA Yastrebov VK, Schaiman MS, Tarasevich IV, Raoult D: Molecular identification of a collection of spotted fever group rickettsiae obtained from patients and ticks from Russia. Am J Trop Med Hyg 2006, 74(3):440-443.

4. Mediannikov OY, Sidelnikov Y, Ivanov L, Mokretsova E, Fournier PE, Tarasevich I, Raoult D: Acute tick-borne rickettsiosis caused by Rickettsia heilongjiangensis in Russian Far East. Emerg Infect Dis 2004, 10(5):810-817.

5. Ando S, Kurosawa M, Sakata A, Fujita H, Sakai K, Sekine M, Katsumi M, Saitou W, Yano Y, Takada N, Takano A, Kawabata H, Hanaoka N, Watanabe H, Kurane I, Kishimoto T: Human Rickettsia heilongjiangensis infection, Japan. Emerg Infect Dis 2010, 16(8):1306-1308.

6. Duan $C$, Meng $Y$, Wang $X$, Xiong $X$, Wen B: Exploratory study on pathogenesis of far-eastern spotted fever. Am J Trop Med Hyg 2011, 85(3):504-509.

7. La Scola B, Raoult D: Laboratory diagnosis of rickettsioses: current approaches to diagnosis of old and new rickettsial diseases. J Clin Microbiol 1997, 35(11):2715.

8. Renvoisé A, Rolain JM, Socolovschi C, Raoult D: Widespread use of realtime PCR for rickettsial diagnosis. FEMS Immuno Med Microbiol 2012, 64(1):126-129.

9. Qi Y, Xiong X, Wang X, Duan C, Jia Y, Jiao J, Gong W, Wen B: Proteome analysis and serological characterization of surface-exposed proteins of Rickettsia heilongjiangensis. PLoS One 2013, 8(7):e70440.

10. Kowalczewska M, Vellaiswamy M, Nappez C, Vincentelli R, Scola BL, Raoult D: Protein candidates for the serodiagnosis of rickettsioses. FEMS Immuno Med Microbiol 2012, 64(1):130-133.

11. Jang WJ, Huh MS, Park KH, Choi MS, Kim IS: Evaluation of an immunoglobulin M capture enzyme-linked immunosorbent assay for diagnosis of Orientia tsutsugamushi infection. Clin Diagn Lab Immunol 2003, 10(3):394-398

12. Chao CC, Huber ES, Porter TB, Zhang Z, Ching WM: Analysis of the crossreactivity of various $56 \mathrm{kDa}$ recombinant protein antigens with serum samples collected after Orientia tsutsugamushi infection by ELISA. Am J Trop Med Hyg 2011, 84(6):967-972.

13. Angkasekwinai N, Atkins EH, Romero S, Grieco J, Chao CC, Ching WM: An evaluation study of enzyme-linked immunosorbent assay (ELISA) using recombinant protein Pap31 for detection of antibody against bartonella bacilliformis infection among the Peruvian population. AmJTrop Med Hyg 2014, 90(4):690-696.

14. Fournier PE, Dumler JS, Greub G, Zhang J, Wu Y, Raoult D: Gene sequencebased criteria for identification of New rickettsia isolates and description of rickettsia heilongjiangensis sp. nov. J Clin Microbiol 2003, 41(12):5456-5465.

15. Qi Y, Xiong X, Duan C, Jiao J, Gong W, Wen B: Recombinant protein YbgF induces protective immunity against Rickettsia heilongjiangensis infection in C3H/HeN mice. Vaccine 2013, 31(48):5643-5650.

16. Xiong $X$, Wang $X$, Wen B, Graves S, Stenos J: Potential serodiagnostic markers for $Q$ fever identified in Coxiella burnetii by immunoproteomic and protein microarray approaches. BMC Microbiol 2012, 12:35.

17. Mezzasoma L, Bacarese-Hamilton T, Di Cristina M, Rossi R, Bistoni F, Crisanti A: Antigen microarrays for serodiagnosis of infectious diseases. Clin Chem 2002, 48(1):121-130.

18. Brouqui P, Bacellar F, Baranton G, Birtles RJ, Bjoersdorff A, Blanco JR, Caruso G, Cinco M, Fournier PE, Francavilla E, Jensenius M, Kazar J, Laferl H, Lakos A, Lotric Furlan S, Maurin M, Oteo JA, Parola P, Perez-Eid C, Peter O, Postic D, Raoult D, Tellez A, Tselentis Y, Wilske B: Guidelines for the diagnosis of tick-borne bacterial diseases in Europe. Clin Microbiol Infect 2004, 10(12):1108-1132.

doi:10.1186/1471-2334-14-332

Cite this article as: Qi et al:: Microarray of surface-exposed proteins of rickettsia heilongjiangensis for serodiagnosis of Far-eastern spotted fever. BMC Infectious Diseases 2014 14:332.

\section{Submit your next manuscript to BioMed Central and take full advantage of:}

- Convenient online submission

- Thorough peer review

- No space constraints or color figure charges

- Immediate publication on acceptance

- Inclusion in PubMed, CAS, Scopus and Google Scholar

- Research which is freely available for redistribution 